De Tampere à Séville : bilan de la sécurité européenne $(1 / 2)$

\title{
Le sommet européen de Laeken 14 - 15 décembre 2001: L'avenir del'Union européenne- Déclaration de Laeken
}

déclaration de Laecken

\section{OpenEdition \\ Journals}

Édition électronique

URL : http://journals.openedition.org/conflits/779

DOI : $10.4000 /$ conflits.779

ISSN : $1777-5345$

Éditeur :

CCLS - Centre d'études sur les conflits lilberté et sécurité, L'Harmattan

Édition imprimée

Date de publication : 1 mars 2002

ISBN : 2-7475-3029-9

ISSN : 1157-996X

Référence électronique

déclaration de Laecken, « Le sommet européen de Laeken 14 - 15 décembre 2001: L'avenir del'Union européenne- Déclaration de Laeken », Cultures \& Conflits [En ligne], 45 I printemps 2002, mis en ligne le 22 mars 2006, consulté le 30 mars 2021. URL : http://journals.openedition.org/conflits/779 ; DOI : https://doi.org/10.4000/conflits.779

Ce document a été généré automatiquement le 30 mars 2021.

Creative Commons License 


\title{
Le sommet européen de Laeken 14 - 15 décembre 2001: L'avenir del'Union européenne- Déclaration de Laeken
}

\author{
déclaration de Laecken
}

\section{L'EUROPE À UN CARREFOUR}

1 Pendant des siècles, des peuples et des Etats ont essayé de s'assurer la maîtrise du continent européen par la guerre et par les armes. Dans ce continent affaibli par deux guerres sanglantes et par le déclin de sa position dans le monde, l'idée que le rêve d'une Europe forte et unie ne pourrait se réaliser que dans la paix et la concertation a fait son chemin. Pour vaincre définitivement les démons du passé, on a commencé par instaurer une communauté du charbon et de l'acier, à laquelle se sont ajoutées par la suite d'autres activités économiques, comme l'agriculture. En fin de compte, un véritable marché unique concernant les marchandises, les personnes, les services et les capitaux a été mis en place, auquel on a adjoint une monnaie unique en 1999. Le 1er janvier 2002, l'euro fera partie de la réalité quotidienne de 300 millions de citoyens européens.

2 L'Union européenne s'est donc créée progressivement. Au début, il s'agissait avant tout d'une coopération économique et technique. Il y a vingt ans, la première élection directe du Parlement européen a considérablement renforcé la légitimité démocratique de la Communauté, dont le Conseil était le seul dépositaire jusque là. Ces dix dernières années, une union politique a été mise en chantier et une coopération a été instaurée dans les domaines de la politique sociale, de l'emploi, de l'asile, de l'immigration, de la police, de la justice, de la politique étrangère, ainsi qu'une politique commune de sécurité et de défense.

3 L'Union européenne est une réussite. Depuis plus d'un demi-siècle, l'Europe vit en paix. Avec l'Amérique du Nord et le Japon, l'Union est l'une des trois régions les plus prospères de la planète. Grâce à la solidarité entre ses membres et à une répartition 
juste des fruits de la croissance économique, le niveau de vie a fortement augmenté dans les régions les plus faibles de l'Union, qui ont rattrapé une grande partie de leur retard.

4 Cinquante ans après sa naissance, l'Union aborde cependant un carrefour, un moment charnière de son existence. L'unification de l'Europe est imminente. L'Union est sur le point de s'ouvrir à plus de dix nouveaux Etats membres, principalement d'Europe centrale et orientale, et à tourner ainsi définitivement une des pages les plus sombres de son histoire, celle de la Seconde Guerre mondiale et du partage artificiel de l'Europe qui l'a suivie. L'Europe va enfin, sans effusion de sang, devenir une grande famille ; il va sans dire que cette véritable mutation demande une autre approche que celle qui a été suivie il y a cinquante ans, lorsque six pays ont lancé le processus.

Le défi démocratique de l'Europe

5 Simultanément, l'Europe est confrontée à un double défi : l'un en son sein, l'autre en dehors de ses frontières.

6 À l'intérieur de l'Union, il faut rapprocher les institutions européennes du citoyen. Certes, les citoyens se rallient aux grands objectifs de l'Union, mais ils ne voient pas toujours le lien entre ces objectifs et l'action quotidienne de l'Union. Ils demandent aux institutions européennes moins de lourdeur et de rigidité et surtout plus d'efficacité et de transparence. Beaucoup trouvent aussi que l'Union doit s'occuper davantage de leurs préoccupations concrètes, plutôt que s'immiscer jusque dans les détails dans des affaires qu'il vaudrait mieux, compte tenu de leur nature, confier aux élus des Etats membres et des régions. Certains ressentent même cette attitude comme une menace pour leur identité. Mais, ce qui est peut-être plus important encore, les citoyens trouvent que tout se règle bien trop souvent à leur insu et veulent un meilleur contrôle démocratique.

Le nouveau rôle de l'Europe dans un environnement mondialisé

7 Hors de ses frontières, l'Union européenne est également confrontée à un environnement en mutation rapide, mondialisé. Après la chute du mur de Berlin, on a cru un moment pouvoir vivre longtemps dans un ordre mondial stable, sans conflits. Les droits de l'homme en constitueraient le fondement. Mais quelques années plus tard, cette certitude a disparu. Le 11 septembre nous a brutalement ouvert les yeux. Les forces contraires n'ont pas disparu ; le fanatisme religieux, le nationalisme ethnique, le racisme et le terrorisme s'intensifient et continuent d'être alimentés par les conflits régionaux, la pauvreté et le sous-développement.

Quel est le rôle de l'Europe dans ce monde transformé ? Maintenant qu'elle est enfin unie, l'Europe ne doit-elle pas jouer un rôle de premier plan dans un nouvel ordre planétaire, celui d'une puissance qui est à même de jouer un rôle stabilisateur au plan mondial et d'être un repère pour un grand nombre de pays et de peuples? L'Europe, continent des valeurs humanistes, de la Magna Carta, du Bill of Rights, de la Révolution française, de la chute du mur de Berlin. Le continent de la liberté, de la solidarité, de la diversité surtout, ce qui implique le respect de la langue, des traditions et de la culture d'autrui. La seule frontière que trace l'Union européenne est celle de la démocratie et des droits de l'homme. L'Union n'est ouverte qu'aux pays qui respectent des valeurs fondamentales telles que des élections libres, le respect des minorités et l'Etat de droit.

9 Maintenant que la guerre froide est terminée et que nous vivons aujourd'hui dans un monde à la fois mondialisé et éclaté, le moment est venu pour l'Europe de prendre ses responsabilités dans la gouvernance de la globalisation. Le rôle qu'elle doit jouer est 
celui d'une puissance qui part résolument en guerre contre toute violence, toute terreur, tout fanatisme, mais qui ne ferme pas les yeux sur les injustices criantes qui existent dans le monde. En résumé, une puissance qui veut faire évoluer les rapports dans le monde de sorte qu'ils produisent des avantages pas uniquement pour les pays riches, mais aussi pour les plus pauvres. Une puissance qui veut encadrer la mondialisation selon les principes de l'éthique, c'est-à-dire l'ancrer dans la solidarité et le développement durable.

Les attentes du citoyen européen

10 L'image d'une Europe démocratique et engagée dans le monde correspond parfaitement aux vœux du citoyen. Celui-ci a fait savoir à maintes reprises qu'il souhaitait que l'Union joue un plus grand rôle dans les domaines de la justice et de la sécurité, de la lutte contre la criminalité transfrontière, de la maitrise des flux migratoires, de l'accueil des demandeurs d'asile et des réfugiés en provenance de zones de conflit périphériques. Il demande aussi des résultats dans le domaine de l'emploi et de la lutte contre la pauvreté et l'exclusion sociale, ainsi que dans le domaine de la cohésion économique et sociale. Il exige une approche commune à l'égard de la pollution, des changements climatiques, de la sûreté des aliments. Bref, à l'égard de tous les problèmes transfrontières dont il sent d'instinct qu'on ne peut y faire face que par la coopération. Tout comme il veut aussi plus d'Europe dans les affaires extérieures, la sécurité et la défense; autrement dit, il demande une action renforcée et mieux coordonnée pour lutter contre les foyers d'incendie qui couvent au sein même et autour de l'Europe et dans le reste du monde.

11 Simultanément, ce même citoyen trouve que l'Union va trop loin et affiche un comportement trop bureaucratique dans nombre d'autres domaines. Le bon fonctionnement du marché intérieur et de la monnaie unique doit rester la pierre angulaire de la coordination de l'environnement économique, financier et fiscal, sans que la spécificité des Etats membres ne soit compromise. Les différences nationales et régionales sont souvent le produit de l'histoire ou de la tradition. Elles peuvent être enrichissantes. En d'autres termes, ce que le citoyen entend par "bonne gestion des affaires publiques", c'est la création de nouvelles opportunités, et non de nouvelles rigidités. Ce qu'il attend, c'est davantage de résultats, de meilleures réponses à des questions concrètes, et pas un "super-Etat" européen ni des institutions européennes qui se mêlent de tout.

12 En résumé, le citoyen demande une approche communautaire claire, transparente, efficace et menée de façon démocratique. Une approche qui fasse de l'Europe un phare pour l'avenir du monde ; une approche qui donne des résultats concrets se traduisant par plus d'emplois, une meilleure qualité de vie, moins de criminalité, une éducation de qualité et de meilleurs soins de santé. Il ne fait pas de doute que l'Europe doive à cette fin se ressourcer et se réformer.

\section{LES DÉFIS ET LES RÉFORMES DANS UNE UNION RENOUVELÉE}

13 L'Union doit devenir plus démocratique, plus transparente et plus efficace. Et elle doit relever trois défis fondamentaux : Comment rapprocher les citoyens, et en premier lieu les jeunes, du projet européen et des institutions européennes? Comment structurer la vie politique et l'espace politique européen dans une Europe élargie ? Comment faire de l'Union un facteur de stabilisation et un repère dans le monde nouveau, multipolaire? Pour trouver des réponses, il faut poser une série de questions ciblées.

Une meilleure répartition et définition des compétences dans l'Union européenne 

respect de l'acquis communautaire, s'il n'y a pas lieu d'ajuster les compétences. Comment les attentes des citoyens peuvent-elles servir de fil conducteur à cette fin? Quelles missions pourraient en résulter pour l'Union? Et, inversement, quelles tâches serait-il préférable de laisser aux Etats membres? Quelles sont les modifications nécessaires à apporter dans le traité aux différentes politiques? Comment formuler, par exemple, une politique extérieure commune et une politique de défense plus cohérentes? Faut-il réactualiser les missions de Petersberg ? Voulons-nous adopter une approche plus intégrée en ce qui concerne la coopération policière et en matière pénale? Comment renforcer la coordination des politiques économiques? Comment pouvons-nous intensifier la coopération dans les domaines de l'insertion sociale, de l'environnement, de la santé, de la sûreté des aliments? Par contre, ne faut-il pas laisser de manière plus explicite la gestion quotidienne et l'exécution de la politique de l'Union aux Etats membres et, là où leur Constitution le prévoit, aux régions? $\mathrm{Ne}$ doivent-ils pas avoir la garantie qu'on ne touchera pas à leurs compétences? compétences ne conduira pas à un élargissement furtif des compétences de l'Union ou qu'elle n'empiétera pas sur les domaines qui relèvent de la compétence exclusive des Etats membres et, le cas échéant, des régions. Comment veiller en même temps à ce que la dynamique européenne ne s'affaiblisse pas? En effet, il faut qu'à l'avenir aussi l'Union soit en mesure de réagir à de nouveaux défis et développements et de sonder de nouveaux domaines d'action. A cette fin, faut-il revoir les articles 95 et 308 du traité, à la lumière de l'acquis jurisprudentiel ?

La simplification des instruments de l'Union

La question de savoir qui fait quoi n'est pas la seule importante ; il importe tout autant de déterminer comment l'Union agit et quels sont les instruments qu'elle utilise. Les modifications successives des traités ont à chaque fois entraîné une prolifération d'instruments. Et les directives ont progressivement évolué pour devenir des actes législatifs de plus en plus détaillés. Il est donc essentiel de se demander si les différents instruments de l'Union ne doivent pas être mieux circonscrits et s'il ne faut pas en réduire le nombre. 
19 En d'autres termes, faut-il introduire une distinction entre mesures législatives et mesures d'exécution? Faut-il réduire le nombre des instruments législatifs : normes directes, législation-cadre et instruments non contraignants (avis, recommandations, coordination ouverte)? Est-il souhaitable ou non de recourir plus souvent aux législations-cadres qui laissent plus de latitude aux Etats membres pour réaliser les objectifs politiques? Pour quelles compétences la coordination ouverte et la reconnaissance mutuelle sont-elles les instruments les plus appropriés? Le principe de proportionnalité reste-t-il le principe de base?

Davantage de démocratie, de transparence et d'efficacité dans l'Union européenne

20 L'Union européenne puise sa légitimité dans les valeurs démocratiques qu'elle véhicule, les objectifs qu'elle poursuit et les compétences et instruments dont elle dispose. Mais le projet européen tire aussi sa légitimité d'institutions démocratiques, transparentes et efficaces. Les parlements nationaux eux aussi contribuent à légitimer le projet européen. La déclaration sur l'avenir de l'Union, annexée au traité de Nice, a souligné la nécessité d'examiner leur rôle dans la construction européenne. Plus généralement, il convient de s'interroger sur les initiatives que nous pouvons prendre pour créer un espace public européen.

21 La première question à se poser est de savoir comment nous pouvons augmenter la légitimité démocratique et la transparence des institutions actuelles, et elle vaut pour les trois institutions.

22 Comment peut-on renforcer l'autorité et l'efficacité de la Commission européenne? Comment doit être désigné le président de la Commission : par le Conseil européen, par le Parlement européen ou par l'élection directe par les citoyens? Faut-il renforcer le rôle du Parlement européen? Faut-il ou non élargir le droit de codécision? Faut-il revoir le mode d'élection des membres du Parlement européen? Convient-il de créer une circonscription électorale européenne ou de maintenir des circonscriptions électorales fixées au niveau national? Peut-on combiner les deux systèmes? Faut-il renforcer le rôle du Conseil ? Le Conseil doit-il intervenir de la même manière quand il agit en tant que législateur et dans le cadre de ses compétences d'exécution? Faut-il pour accroître la transparence, que les sessions du Conseil soient publiques, en tout cas lorsque le Conseil agit en sa capacité de législateur? Le citoyen doit-il avoir plus d'accès aux documents du Conseil ? Enfin, comment garantir l'équilibre et le contrôle mutuel entre les institutions?

Une deuxième question, qui a également trait à la légitimité démocratique, concerne le rôle des parlements nationaux. Doivent-ils être représentés dans une nouvelle institution, à côté du Conseil et du Parlement européen? Doivent-ils jouer un rôle dans les domaines de l'action européenne pour lesquels le Parlement européen n'est pas compétent? Doivent-ils se concentrer sur la répartition des compétences entre l'Union et les Etats membres, par exemple par un contrôle préalable du respect du principe de subsidiarité?

La troisième question à se poser porte sur les moyens d'améliorer l'efficacité du processus décisionnel et le fonctionnement des institutions dans une Union de quelque trente Etats membres. Comment l'Union pourrait-elle mieux fixer ses objectifs et ses priorités et en assurer une meilleure mise en œuvre? Faut-il davantage de décisions prises à la majorité qualifiée ? Comment simplifier et accélérer la procédure de codécision entre le Conseil et le Parlement européen? Peut-on garder la rotation semestrielle de la présidence de l'Union? Quel sera le rôle futur du Parlement 
européen? Qu'adviendra-t-il à l'avenir du rôle et de la structure des différentes formations du Conseil ? Comment accroître par ailleurs la cohérence de la politique étrangère européenne? Comment renforcer la synergie entre le Haut Représentant et le Commissaire compétent pour ces questions? Devons-nous renforcer encore la représentation de l'Union dans les enceintes internationales?

La voie vers une Constitution pour les citoyens européens

L'Union européenne fonctionne actuellement avec quatre traités. Les objectifs, les compétences et les instruments politiques de l'Union se trouvent dispersés dans l'ensemble de ces traités. Si l'on veut plus de transparence, une simplification est indispensable.

Quatre séries de questions peuvent être posées à cet égard. La première concerne la simplification des traités actuels sans en changer le contenu. Faut-il revoir la distinction entre l'Union et les Communautés ? Que faire de la division en trois piliers?

Il faut ensuite réfléchir à un éventuel réaménagement des traités. Faut-il faire une distinction entre un traité de base et les autres dispositions des traités? Cette distinction doit-elle être concrétisée par une scission des textes ? Cela peut-il conduire à faire une distinction entre les procédures de modification et de ratification pour le traité de base et les autres dispositions des traités?

Il faut ensuite se demander si la Charte des droits fondamentaux doit être intégrée dans le traité de base et se poser la question de l'adhésion de la Communauté européenne à la Convention européenne des droits de l'homme.

Se pose enfin la question de savoir si cette simplification et ce réaménagement ne devraient pas conduire à terme à l'adoption d'un texte constitutionnel. Quels devraient être les éléments essentiels d'une telle Constitution? Les valeurs auxquelles l'Union est attachée, les droits fondamentaux et les devoirs des citoyens, les relations des Etats membres dans l'Union?

\section{LA CONVOCATION D'UNE CONVENTION SUR L'AVENIR DE L'EUROPE}

Pour assurer une préparation aussi large et aussi transparente que possible de la prochaine Conférence intergouvernementale, le Conseil européen a décidé de convoquer une Convention rassemblant les principales parties prenantes au débat sur l'avenir de l'Union. Compte tenu de ce qui précède, cette Convention aura pour tâche d'examiner les questions essentielles que soulève le développement futur de l'Union et de rechercher les différentes réponses possibles.

31 Le Conseil européen a désigné M.V. Giscard d'Estaing comme Président de la Convention et MM. G. Amato et J.L. Dehaene comme Vice-Présidents.

Composition

32 Outre son Président et ses deux Vice-Présidents, la Convention sera composée de 15 représentants des chefs d'Etat ou de gouvernement des Etats membres (1 par Etat membre), de 30 membres des parlements nationaux ( 2 par Etat membre), de 16 membres du Parlement européen et de deux représentant de la Commission. Les pays candidats à l'adhésion participeront à part entière aux travaux de la Convention. Ils seront représentés dans les mêmes conditions que les Etats membres actuels (un représentant $\mathrm{du}$ gouvernement et deux membres $\mathrm{du}$ parlement national) et participeront aux délibérations sans toutefois pouvoir empêcher le consensus qui se dégagerait entre les Etats membres. 
Les membres de la Convention ne pourront se faire remplacer par leurs suppléants que s'ils sont absents. Les membres suppléants seront désignés de la même manière que les membres effectifs.

Le Présidium de la Convention sera composé du Président de la Convention, des deux Vice-Présidents de la Convention et de neuf membres issus de la Convention (les représentant de tous les gouvernements qui pendant la Convention exercent la présidence du Conseil, deux représentants des parlements nationaux, deux représentants des membres du Parlement européen et deux représentants de la Commission.

Seront invités comme observateurs trois représentants du Comité économique et social et trois représentants des partenaires sociaux européens, auxquels s'ajouteront, au nom du Comité des régions, six représentants (à désigner par le Comité des régions parmi les régions, les villes et les régions dotées de pouvoirs législatifs), ainsi que le médiateur européen. Le Président de la Cour de Justice et celui de la Cour des comptes pourront s'exprimer devant la Convention à l'invitation du Présidium.

Durée des travaux

6 La Convention tiendra sa séance inaugurale le 1er mars 2002. A cette occasion, elle procédera à la désignation de son Présidium et arrêtera ses méthodes de travail. Les travaux s'achèveront après une année, à temps pour permettre au Président de la Convention d'en présenter les résultats au Conseil européen.

Méthodes de travail

7 Le Président préparera le début des travaux de la Convention en tirant les enseignements du débat public. Le Présidium aura un rôle d'impulsion et fournira une première base de travail pour la Convention.

Le Présidium pourra consulter les services de la Commission et les experts de son choix sur toute question technique qu'il jugerait utile d'approfondir. Il pourra créer des groupes de travail ad hoc.

Le Conseil se tiendra informé de l'état d'avancement des travaux de la Convention. Le Président de la Convention présentera un rapport oral sur l'état d'avancement des travaux à chaque Conseil européen, ce qui permettra, par la même occasion, de recueillir le sentiment des Chefs d'Etat ou de gouvernement.

40 La Convention se réunira à Bruxelles. Les débats de la Convention et l'ensemble des documents officiels seront publics. La Convention travaillera dans les onze langues de travail de l'Union.

Document final

41 La Convention étudiera les différentes questions. Elle établira un document final qui pourra comprendre soit différentes options, en précisant le soutien qu'elles ont recueilli, soit des recommandations en cas de consensus.

42 Avec le résultat des débats nationaux sur l'avenir de l'Union, le document final servira de point de départ pour les discussions de la Conférence intergouvernementale, qui prendra les décisions définitives.

Forum

3 Pour élargir le débat et y associer l'ensemble des citoyens, un Forum sera ouvert aux organisations représentant la société civile (partenaires sociaux, milieux économiques, organisations non gouvernementales, milieux académiques, etc.). Il s'agira d'un réseau 
structuré d'organisations qui seront régulièrement informées des travaux de la Convention. Leurs contributions seront versées au débat. Ces organisations pourront être auditionnées ou consultées sur des sujets particuliers selon des modalités à déterminer par le Présidium.

Secrétariat

Le Présidium sera assisté par un Secrétariat de la Convention, qui sera assuré par le Secrétariat général du Conseil. Des experts de la Commission et du Parlement européen pourront en faire partie.

INDEX

Mots-clés : construction européenne, textes et documents officiels 